\title{
Extended IOWG Operator and its Use in Group Decision Making Based on Multiplicative Linguistic Preference Relations
}

\author{
Zeshui Xu \\ Institute of Sciences, PLA University of Science and Technology \\ Nanjing, Jiangsu 210007, China
}

\begin{abstract}
In [1], Xu and Da introduced the Induced Ordered Weighted Geometric (IOWG) operator, which takes as its argument pairs, called OWG pairs, in which one component is used to induce an ordering over the second components which are exact numerical values and then aggregated. In this study, we develop an extended IOWG (EIOWG) operator, in which the second components are linguistic variables. We study some desirable properties of the EIOWG operator, and then apply the EIOWG operator to group decision making based on multiplicative linguistic preference relations.
\end{abstract}

Keywords: Aggregation, Induced Ordered Weighted Geometric (IOWG) Operator, Group Decision Making, Multiplicative Linguistic Preference Relation

\section{INTRODUCTION}

The ordered weighted averaging (OWA) operator was developed by Yager [2]. The fundamental aspect of the OWA operator is a reordering step in which the input arguments are rearranged in descending order [2-8]. The ordered weighted geometric (OWG) operator is an aggregation operator that is based on the OWA operator and the geometric mean [1,9-15]. Yager and Filev [16] introduced a more general type of OWA operator called induced ordered weighted averaging (IOWA) operator. The IOWA operator takes as its argument pairs, called OWA pairs, in which one component is used to induce an ordering over the second components which are exact numerical values and then aggregated. Recently, $\mathrm{Xu}$ and $\mathrm{Da}$ [1] developed an induced ordered weighted geometric (IOWG) operator that is based on the IOWA operator and the geometric mean, which can be used to aggregate multiplicative preference relations with exact numerical values in group decision making problems [17]. However, in many situations, the input arguments take the form of linguistic variables rather than numerical ones $[13,18-38]$. Therefore, it is necessary to pay attention to this issue. In this study, we shall develop an extended IOWG (EIOWG) operator, and study some desirable properties of the EIOWG operator. Then, we shall develop an approach, based on the EIOWG and the extended OWG (EOWG) operators, for ranking alternatives in group decision making with multiplicative linguistic preference relations. Finally, we shall apply the developed approach to the evaluation of investment alternatives of an investment company and draw our conclusions.
EIOWG Operator: Let $S=\left\{s_{\alpha} \mid \alpha=1 / t, \ldots, 1 / 2,1,2, \ldots, t\right\}$ be a multiplicative linguistic term set with odd cardinality. Any label, $s_{\alpha}$, represents a possible value for a linguistic variable, and it should satisfy the following characteristics:

1. The set is ordered: $s_{\alpha}>s_{\beta}$ if $\alpha>\beta$;

2. There is the reciprocal operator: $\operatorname{rec}\left(s_{\alpha}\right)=s_{\beta}$ such that $\alpha \beta=1$.

We call this multiplicative linguistic term set $S$ the multiplicative linguistic scale. For example, $S$ can be defined as:

$S=\left\{s_{1 / 5}=\right.$ extremely low, $s_{1 / 4}=$ very low, $s_{1 / 3}=$ low, $s_{1 / 2}=$ slightly low, $s_{1}=$ medium, $s_{2}=$ slightlyhigh,

$s_{3}=$ high,$s_{4}=$ very high, $s_{5}=$ extremely high $\}$

To preserve all the given information, we extend the discrete multiplicative linguistic term set $S$ to a continuous multiplicative linguistic term set $\bar{S}=\left\{s_{\alpha} \mid \alpha \in[1 / q, q]\right\}$, where $q(q>t)$ is a sufficiently large positive integer. If $s_{\alpha} \in S$, then we call $s_{\alpha}$ the original linguistic term, otherwise, we call $s_{\alpha}$ the virtual linguistic term. In general, the decision maker uses the original linguistic terms to evaluate alternatives, and the virtual linguistic terms can only appear in calculation.

Let $s_{\alpha}, s_{\beta} \in \bar{S}$, and $\mu, \mu_{1}, \mu_{2} \in[0,1]$, we give

some operational laws as follows [37]: 
1. $\left(s_{\alpha}\right)^{\mu}=s_{\alpha^{\mu}} ;$

2. $\left(s_{\alpha}\right)^{\mu_{1}} \otimes\left(s_{\alpha}\right)^{\mu_{2}}=\left(s_{\alpha}\right)^{\mu_{1}+\mu_{2}}$;

3. $\left(s_{\alpha} \otimes s_{\beta}\right)^{\mu}=\left(s_{\alpha}\right)^{\mu} \otimes\left(s_{\beta}\right)^{\mu}$;

4. $s_{\alpha} \otimes s_{\beta}=s_{\beta} \otimes s_{\alpha}=s_{\alpha \beta}$.

The ordered weighted geometric (OWG) operator is an aggregation operator that Chiclana et al. [9] defined and characterized to design multiplicative decision-making models [10,11,14]. It is based on the ordered weighted averaging (OWA) operator [2] and on the geometric mean. Xu and Da [12] presented some families of OWG operators.

Definition 1[1,9-15]: An OWG operator of dimension $n$ is a mapping $O W G: R^{+^{n}} \rightarrow R^{+}$which has associated with it an exponential weighting vector $w=\left(w_{1}, w_{2}, \ldots, w_{n}\right)^{T}$, with $w_{j} \in[0,1]$ and

$\sum_{j=1}^{n} w_{j}=1$, such that

$O W G_{w}\left(\alpha_{1}, \alpha_{2} \ldots, \alpha_{n}\right)=\prod_{j=1}^{n} b_{j}^{w_{j}}$

where $b_{j}$ is the $j$ th largest of the $\alpha_{i}, R^{+}$is the positive real number set.

The OWG operator has only been used in situations in which the input arguments are the exact numerical values. However, judgements of people depend on personal psychological aspects such as experience, learning, situation, state of mind, and so forth. It is more suitable to provide their preferences by means of linguistic variables rather than numerical ones (for example when evaluating the comfort or design of a car, terms like good, fair, poor can be used). In [37], Xu extended the OWG operator to accommodate the situations where the input arguments are linguistic variables.

Definition 2 [37]: An extended ordered weighted geometric (EOWG) operator of dimension $n$ is a mapping $E O W G: \bar{S}^{n} \rightarrow \bar{S}_{n}$, which has associated with it an exponential weighting vector $w=\left(w_{1}, w_{2}, \ldots, w_{n}\right)^{T}$, with $w_{j} \in[0,1]$ and $\sum_{j=1}^{n} w_{j}=1$, such that;

$\begin{aligned} E O W G_{w}\left(s_{\alpha_{1}}, s_{\alpha_{2}}, \ldots, s_{\alpha_{n}}\right) & =\left(s_{\beta_{1}}\right)^{w_{1}} \otimes\left(s_{\beta_{2}}\right)^{w_{2}} \otimes \ldots \otimes\left(s_{\beta_{n}}\right)^{w_{n}} \\ & =\left(s_{\beta_{1}^{w_{1}}}\right) \otimes\left(s_{\beta_{2}^{w_{2}}}\right) \otimes \cdots \otimes\left(s_{\beta_{n}^{w_{n}}}\right)^{n} \\ & =s_{\bar{\beta}}\end{aligned}$

where $\beta=\prod_{j=1}^{n} \beta_{j}^{w_{j}}, s_{\beta_{j}}$ is the $j$ th largest of the $s_{\alpha_{j}}$, which is an extension of the OWG operator.

Example 1: Assume $w=(0.3,0.2,0.4,0.1)^{T}$, then

$$
\begin{aligned}
\operatorname{EOWG}_{w}\left(s_{1 / 2}, s_{4}, s_{1 / 3}, s_{5}\right) & =\left(s_{5}\right)^{0.3} \otimes\left(s_{4}\right)^{0.2} \otimes\left(s_{1 / 2}\right)^{0.4} \otimes\left(s_{1 / 3}\right)^{0.1} \\
& =\left(s_{5^{0.3}}\right) \otimes\left(s_{4^{0.1}}\right) \otimes\left(s_{(1 / 2)^{0.4}}\right) \otimes\left(s_{(1 / 3)^{0.2}}\right) \\
& =s_{1.45}
\end{aligned}
$$

In [2], Yager defined the concept of the ordered weighted averaging (OWA) operator. Later, Yager and Filev [16] introduced a more general type of OWA operator called induced ordered weighted averaging (IOWA) operator. The 
IOWA operator takes as its argument pairs, called OWA pairs, in which one component is used to induce an ordering over the second components which are exact numerical values and then aggregated. $\mathrm{Xu}$ and $\mathrm{Da}$ [1] developed an induced ordered weighted geometric (IOWG) operator that is based on the IOWA operator and the geometric mean, which can be used to aggregate multiplicative preference relations with exact numerical values in group decision-making problems.

Definition 3 [1]: An IOWG operator is defined as follows:

$I O W G_{w}\left(\left\langle u_{1}, a_{1}\right\rangle,\left\langle u_{2}, a_{2}\right\rangle \ldots,\left\langle u_{n}, a_{n}\right\rangle\right)=\prod_{j=1}^{n} b_{j}^{w_{j}}$

where $w=\left(w_{1}, w_{2}, \ldots, w_{n}\right)^{T}$ is an exponential weighting vector, such that $w_{j} \in[0,1], \sum^{n} w_{j}=1, b_{j}$ is the $a_{i}$ value of the OWG pair $\left\langle u_{i}, a_{i}\right\rangle$ having the $j$ th largest $u_{i}$, and $u_{i}$ in $\left\langle u_{i}, a_{i}\right\rangle$ is =referred to as the order inducing variable and $a_{i}$ as the argument variable, $a_{i} \in R^{+}, i=1,2, \ldots, n, R^{+}$is the real set. Especially, if $w=(1 / n, 1 / n, \ldots, 1 / n)^{T}$, then $I O W G$ is reduced to the geometric mean operator; if $u_{i}=a_{i}$, for all $i$, then $I O W G$ is reduced to the OWG operator; if $u_{i}=$ No. $i$, for all $i$, where No. $i$ is the ordered position of the $a_{i}$, then $I O W G$ is the weighted geometric mean operator.

In the following, we shall extend the IOWG operator to accommodate the situations where the input arguments are linguistic variables.

Definition 4: An extended IOWG (EIOWG) operator is defined as follows:

$\operatorname{EIOWG}_{w}\left(\left\langle u_{1}, s_{\alpha_{1}}\right\rangle,\left\langle u_{2}, s_{\alpha_{2}}\right\rangle \ldots,\left\langle u_{n}, s_{\alpha_{n}}\right\rangle\right)=\left(s_{\gamma_{1}}\right)^{w_{1}} \otimes\left(s_{\gamma_{2}}\right)^{w_{2}} \otimes \ldots \otimes\left(s_{\gamma_{n}}\right)^{w_{n}}=s_{\bar{\gamma}}$

where $\bar{\gamma}=\prod_{j=1}^{n} \gamma_{j}^{w_{j}}, w=\left(w_{1}, w_{2}, \ldots, w_{n}\right)^{T}$ is an exponential weighting vector, such that $w_{j} \in[0,1], \sum_{\mathrm{j}=1}^{\mathrm{n}} \mathrm{w}_{\mathrm{j}}=1$, $s_{\gamma_{j}}$ is the $s_{\alpha_{i}}$ value of the OWG pair $\left\langle u_{i}, s_{\alpha_{i}}\right\rangle$ having the $j$ th largest $u_{i}$, and $u_{i}$ in $\left\langle u_{i}, s_{\alpha_{i}}\right\rangle$ is referred to as the order inducing variable and $s_{\alpha_{i}}$ as the multiplicative linguistic argument variable. Especially, if $w=(1 / n, 1 / n, \ldots, 1 / n)^{T}$, then $E I O W G$ is reduced to the extended geometric mean operator; if $u_{i}=s_{\alpha_{i}}$, for all $i$, then EIOWG is reduced to the EOWG operator; if $u_{i}=N o$. $i$, for all $i$, where No. $i$ is the ordered position of the $s_{i}$, then $E I O W G$ is reduced to the extended weighted geometric mean operator.

Example 2: Consider a collection of four OWG pairs $\left\langle\right.$ No.1, $\left.s_{1 / 3}\right\rangle,\left\langle N o .3, s_{4}\right\rangle,\left\langle N o .4, s_{1 / 2}\right\rangle$, and $\left\langle N o .2, s_{5}\right\rangle$, we desire to aggregate using the weighting vector $w=(0.2,0.3,0.3,0.2)^{T}$. Performing the ordering the OWG pairs with respect to the first component, we get

$$
\left\langle\text { No. } 1, s_{1 / 3}\right\rangle,\left\langle\text { No.2, } s_{5}\right\rangle,\left\langle N o .3, s_{4}\right\rangle,\left\langle N o .4, s_{1 / 2}\right\rangle
$$

This ordering induces the ordered linguistic arguments

$$
s_{\gamma_{1}}=s_{1 / 3}, s_{\gamma_{2}}=s_{5}, s_{\gamma_{3}}=s_{4}, s_{\gamma_{4}}=s_{1 / 2}
$$

and from this, we get an aggregated value

$$
E I O W G_{w}\left(\left\langle N o .1, s_{1 / 3}\right\rangle,\left\langle N o .3, s_{4}\right\rangle,\left\langle N o .4, s_{1 / 2}\right\rangle,\left\langle N o .2, s_{5}\right\rangle\right)=\left(s_{1 / 3}\right)^{0.2} \otimes\left(s_{5}\right)^{0.3} \otimes\left(s_{4}\right)^{0.3} \otimes\left(s_{1 / 2}\right)^{0.2}
$$




$$
=S_{(1 / 3)^{0.2}} \otimes S_{5^{0.3}} \otimes S_{4^{0.3}} \otimes S_{(1 / 2)^{0.2}}=s_{1.72}
$$

Example 3: Consider the following collection of OWG pairs $\left\langle 0.3, s_{1 / 4}\right\rangle,\left\langle 0.1, s_{5}\right\rangle,\left\langle 0.4, s_{1 / 2}\right\rangle,\left\langle 0.6, s_{3}\right\rangle$ Performing the ordering the OWG pairs with respect to the first component, we have $\left\langle 0.6, s_{3}\right\rangle,\left\langle 0.4, s_{1 / 2}\right\rangle,\left\langle 0.3, s_{1 / 4}\right\rangle$,

This ordering induces the ordered linguistic arguments $s_{\gamma_{1}}=s_{3}, s_{\gamma_{2}}=s_{1 / 2}, s_{\gamma_{3}}=s_{1 / 4}, s_{\gamma_{4}}=s_{5}$ If the weighting vector $w=(0.1,0.4,0.3,0.2)^{T}$, then we get an aggregated value

$$
\begin{aligned}
& \operatorname{EIOWG}_{w}\left(\left\langle 0.3, s_{1 / 4}\right\rangle,\left\langle 0.1, s_{5}\right\rangle,\left\langle 0.4, s_{1 / 2}\right\rangle,\left\langle 0.6, s_{3}\right\rangle\right)=\left(s_{3}\right)^{0.1} \otimes\left(s_{1 / 2}\right)^{0.4} \otimes\left(s_{1 / 4}\right)^{0.3} \otimes\left(s_{5}\right)^{0.2} \\
& =s_{3^{0.1}} \otimes s_{(1 / 2)^{0.4}} \otimes s_{(1 / 4)^{0.3}} \otimes s_{5^{0.2}}=s_{0.77}
\end{aligned}
$$

However, if we replace the objects in Example 3 with $\left\langle 0.1, s_{1 / 4}\right\rangle,\left\langle 0.1, s_{5}\right\rangle,\left\langle 0.4, s_{1 / 2}\right\rangle,\left\langle 0.6, s_{3}\right\rangle$

then there is a tie between $\left\langle 0.1, s_{1 / 4}\right\rangle$ and $\left\langle 0.1, s_{5}\right\rangle$ with respect to order inducing variable. In this case, we can follow the policy presented by Yager and Filev [16], that is, to replace the arguments of the tied objects by the average of the arguments of the tied objects. Thus, for Example 3, we replace the argument component of each of $\left\langle 0.1, s_{1 / 4}\right\rangle$ and $\left\langle 0.1, s_{5}\right\rangle$ by their geometric mean $\left(s_{1 / 4} \otimes s_{5}\right)^{1 / 2}=s_{1.12}$. This substitution gives us ordered linguistic arguments $s_{\gamma_{1}}=s_{3}, s_{\gamma_{2}}=s_{1 / 2}, s_{\gamma_{3}}=s_{1.12}, s_{\gamma_{4}}=s_{1.12}$ thus $\operatorname{EIOWG}_{\mathrm{w}}\left(\left\langle 0.1, \mathrm{~s}_{1 / 4}\right\rangle,\left\langle 0.1, \mathrm{~s}_{5}\right\rangle,\left\langle 0.4, \mathrm{~s}_{1 / 2}\right\rangle,\left\langle 0.6, \mathrm{~s}_{3}\right\rangle\right)=\left(s_{3}\right)^{0.1} \otimes\left(s_{1 / 2}\right)^{0.4} \otimes\left(s_{1.12}\right)^{0.3} \otimes\left(s_{1.12}\right)^{0.2}$

$$
=S_{3^{0.1}} \otimes S_{(1 / 2)^{0.4}} \otimes S_{1.12^{0.3}} \otimes S_{1.12^{0.3}}=s_{0.91}
$$

If $k$ items are tied, we replace these by $k$ replicas of their geometric mean. In the following, we shall study some desirable properties of the EIOWG operator.

\section{Theorem 1 (Commutativity):}

$\operatorname{EIOWG}_{w}\left(\left\langle u_{1}, s_{\alpha_{1}}\right\rangle,\left\langle u_{2}, s_{\alpha_{2}}\right\rangle, \ldots,\left\langle u_{n}, s_{\alpha_{n}}\right\rangle\right)=\operatorname{EIOWG}_{w}\left(\left\langle u_{1}^{\prime}, s_{\alpha_{1}}^{\prime}\right\rangle,\left\langle u_{2}^{\prime}, s_{\alpha_{2}}^{\prime}\right\rangle, \ldots,\left\langle u_{n}^{\prime}, s_{\alpha_{n}}^{\prime}\right\rangle\right)$

$\operatorname{EIOWG}_{w}\left(\left\langle u_{1}, s_{\alpha_{1}}\right\rangle,\left\langle u_{2}, s_{\alpha_{2}}\right\rangle, \ldots,\left\langle u_{n}, s_{\alpha_{n}}\right\rangle\right)=\operatorname{EIOWG}_{w}\left(\left\langle u_{1}^{\prime}, s_{\alpha_{1}}^{\prime}\right\rangle,\left\langle u_{2}^{\prime}, s_{\alpha_{2}}^{\prime}\right\rangle, \ldots,\left\langle u_{n}^{\prime}, s_{\alpha_{n}}^{\prime}\right\rangle\right)$

where $\left(\left\langle u_{1}^{\prime}, s_{\alpha_{1}}^{\prime}\right\rangle,\left\langle u_{2}^{\prime}, s_{\alpha_{2}}^{\prime}\right\rangle, \ldots,\left\langle u_{n}^{\prime}, s_{\alpha_{n}}^{\prime}\right\rangle\right)$ is any permutation of $\left(\left\langle u_{1}, s_{\alpha_{1}}\right\rangle,\left\langle u_{2}, s_{\alpha_{2}}\right\rangle, \ldots,\left\langle u_{n}, s_{\alpha_{n}}\right\rangle\right)$.

Proof. Let

$$
\operatorname{EIOWG}_{w}\left(\left\langle u_{1}, s_{\alpha_{1}}\right\rangle,\left\langle u_{2}, s_{\alpha_{2}}\right\rangle, \ldots,\left\langle u_{n}, s_{\alpha_{n}}\right\rangle\right)=\left(s_{\gamma_{1}}\right)^{w_{1}} \otimes\left(s_{\gamma_{2}}\right)^{w_{2}} \otimes \ldots \otimes\left(s_{\gamma_{n}}\right)^{w_{n}}
$$


$\operatorname{EIOWG}_{w}\left(\left\langle u_{1}^{\prime}, s_{\alpha_{1}}^{\prime}\right\rangle,\left\langle u_{2}^{\prime}, s_{\alpha_{2}}^{\prime}\right\rangle, \ldots,\left\langle u_{n}^{\prime}, s_{\alpha_{n}}^{\prime}\right\rangle\right)=\left(s_{\gamma_{1}}^{\prime}\right)^{w_{1}} \otimes\left(s_{\gamma_{2}}^{\prime}\right)^{w_{2}} \otimes \ldots \otimes\left(s_{\gamma_{n}}^{\prime}\right)^{w_{n}}$

Since $\left(\left\langle u_{1}^{\prime}, s_{\alpha_{1}}^{\prime}\right\rangle,\left\langle u_{2}^{\prime}, s_{\alpha_{2}}^{\prime}\right\rangle, \ldots,\left\langle u_{n}^{\prime}, s_{\alpha_{n}}^{\prime}\right\rangle\right)$ is a permutation of $\left(\left\langle u_{1}, s_{\alpha_{1}}\right\rangle,\left\langle u_{2}, s_{\alpha_{2}}\right\rangle, \ldots,\left\langle u_{n}, s_{\alpha_{n}}\right\rangle\right)$, we have $s_{\gamma_{j}}=s_{\gamma_{j}}^{\prime}(j=1,2, \ldots, n)$, then

$\operatorname{EIOWG}_{w}\left(\left\langle u_{1}, s_{\alpha_{1}}\right\rangle,\left\langle u_{2}, s_{\alpha_{2}}\right\rangle, \ldots,\left\langle u_{n}, s_{\alpha_{n}}\right\rangle\right)=\operatorname{EIOWG}_{w}\left(\left\langle u_{1}^{\prime}, s_{\alpha_{1}}^{\prime}\right\rangle,\left\langle u_{2}^{\prime}, s_{\alpha_{2}}^{\prime}\right\rangle, \ldots,\left\langle u_{n}^{\prime}, s_{\alpha_{n}}^{\prime}\right\rangle\right)$

Theorem 2 (Idempotency): If $s_{\alpha_{j}}=s_{\alpha}$, for all $j$, then

$\operatorname{EIOWG}_{w}\left(\left\langle u_{1}, s_{\alpha_{1}}\right\rangle,\left\langle u_{2}, s_{\alpha_{2}}\right\rangle, \ldots,\left\langle u_{n}, s_{\alpha_{n}}\right\rangle\right)=s_{\alpha}$

Proof: Since $s_{\alpha_{j}}=s_{\alpha}$, for all $j$, we have

$\operatorname{EIOWG}_{w}\left(\left\langle u_{1}, s_{\alpha_{1}}\right\rangle,\left\langle u_{2}, s_{\alpha_{2}}\right\rangle, \ldots,\left\langle u_{n}, s_{\alpha_{n}}\right\rangle\right)=\left(s_{\gamma_{1}}\right)^{w_{1}} \otimes\left(s_{\gamma_{2}}\right)^{w_{2}} \otimes \ldots \otimes\left(s_{\gamma_{n}}\right)^{w_{n}}$

$=\left(s_{\alpha}\right)^{w_{1}} \otimes\left(s_{\alpha}\right)^{w_{2}} \otimes \cdots \otimes\left(s_{\alpha}\right)^{w_{n}}$

$=\left(s_{\alpha}\right)^{\sum_{j=1}^{n} w_{j}}=s_{\alpha}$

Theorem 3 (Monotonicity): If $s_{\alpha_{j}} \leq \hat{s}_{\alpha_{j}}$, for all $j$, then

$\operatorname{EIOWG}_{w}\left(\left\langle u_{1}, s_{\alpha_{1}}\right\rangle,\left\langle u_{2}, s_{\alpha_{2}}\right\rangle, \ldots,\left\langle u_{n}, s_{\alpha_{n}}\right\rangle\right) \leq \operatorname{EIOWG}_{w}\left(\left\langle u_{1}, \hat{s}_{\alpha_{1}}\right\rangle,\left\langle u_{2}, \hat{s}_{\alpha_{2}}\right\rangle, \ldots,\left\langle u_{n}, \hat{s}_{\alpha_{n}}\right\rangle\right)$

Proof: Let

$\operatorname{EIOWG}_{w}\left(\left\langle u_{1}, s_{\alpha_{1}}\right\rangle,\left\langle u_{2}, s_{\alpha_{2}}\right\rangle, \ldots,\left\langle u_{n}, s_{\alpha_{n}}\right\rangle\right)=\left(s_{\gamma_{1}}\right)^{w_{1}} \otimes\left(s_{\gamma_{2}}\right)^{w_{2}} \otimes \ldots \otimes\left(s_{\gamma_{n}}\right)^{w_{n}}$

$\operatorname{EIOWG}_{w}\left(\left\langle u_{1}, \hat{s}_{\alpha_{1}}\right\rangle,\left\langle u_{2}, \hat{s}_{\alpha_{2}}\right\rangle, \ldots,\left\langle u_{n}, \hat{s}_{\alpha_{n}}\right\rangle\right)=\left(\hat{s}_{\gamma_{1}}\right)^{w_{1}} \otimes\left(\hat{s}_{\gamma_{2}}\right)^{w_{2}} \otimes \ldots \otimes\left(\hat{s}_{\gamma_{n}}\right)^{w_{n}}$

Since $s_{\alpha_{j}} \leq \hat{s}_{\alpha_{j}}$, for all $j$, it follows that $s_{\gamma_{j}} \leq \hat{s}_{\gamma_{j}}$, then

$\operatorname{EIOWG}_{w}\left(\left\langle u_{1}, s_{\alpha_{1}}\right\rangle,\left\langle u_{2}, s_{\alpha_{2}}\right\rangle, \ldots,\left\langle u_{n}, s_{\alpha_{n}}\right\rangle\right) \leq \operatorname{EIOWG}_{w}\left(\left\langle u_{1}, \hat{s}_{\alpha_{1}}\right\rangle,\left\langle u_{2}, \hat{s}_{\alpha_{2}}\right\rangle, \ldots,\left\langle u_{n}, \hat{s}_{\alpha_{n}}\right\rangle\right)$

Theorem 4 (Bounded): $\operatorname{Min}_{j}\left(s_{\alpha_{j}}\right) \leq E I O W G_{w}\left(\left\langle u_{1}, s_{\alpha_{1}}\right\rangle,\left\langle u_{2}, s_{\alpha_{2}}\right\rangle, \ldots,\left\langle u_{n}, s_{\alpha_{n}}\right\rangle\right) \leq \operatorname{Max}_{j}\left(s_{\alpha_{j}}\right)$

Proof: Let $\operatorname{Max}_{j}\left(s_{\alpha_{j}}\right)=s_{\beta}$ and $\operatorname{Min}_{j}\left(s_{\alpha_{j}}\right)=s_{\alpha}$, then

$\operatorname{EIOWG}_{w}\left(\left\langle u_{1}, s_{\alpha_{1}}\right\rangle,\left\langle u_{2}, s_{\alpha_{2}}\right\rangle, \ldots,\left\langle u_{n}, s_{\alpha_{n}}\right\rangle\right)=\left(s_{\gamma_{1}}\right)^{w_{1}} \otimes\left(s_{\gamma_{2}}\right)^{w_{2}} \otimes \cdots \otimes\left(s_{\gamma_{n}}\right)^{w_{n}}$

$\leq\left(s_{\beta}\right)^{w_{1}} \otimes\left(s_{\beta}\right)^{w_{2}} \otimes \cdots \otimes\left(s_{\beta}\right)^{w_{n}}=\left(s_{\beta}\right)^{\sum_{j=1}^{n} w_{j}}=s_{\beta}$ 
$\operatorname{EIOWG}_{w}\left(\left\langle u_{1}, s_{\alpha_{1}}\right\rangle,\left\langle u_{2}, s_{\alpha_{2}}\right\rangle, \ldots,\left\langle u_{n}, s_{\alpha_{n}}\right\rangle\right)=\left(s_{\gamma_{1}}\right)^{w_{1}} \otimes\left(s_{\gamma_{2}}\right)^{w_{2}} \otimes \ldots \otimes\left(s_{\gamma_{n}}\right)^{w_{n}}$

$\geq\left(s_{\alpha}\right)^{w_{1}} \otimes\left(s_{\alpha}\right)^{w_{2}} \otimes \cdots \otimes\left(s_{\alpha}\right)^{w_{n}}=\left(s_{\alpha}\right)^{\sum_{j=1}^{n} w_{j}}=s_{\alpha}$

hence $\operatorname{Min}_{j}\left(s_{\alpha_{j}}\right) \leq \operatorname{EIOWG}_{w}\left(\left\langle u_{1}, s_{\alpha_{1}}\right\rangle,\left\langle u_{2}, s_{\alpha_{2}}\right\rangle, \ldots,\left\langle u_{n}, s_{\alpha_{n}}\right\rangle\right) \leq \underset{j}{\operatorname{Max}}\left(s_{\alpha_{j}}\right)$

An Approach Based on the EIOWG and the EOWG Operators to Group Decision Making with Multiplicative Linguistic Preference Relations: Consider a group decision making problem with linguistic preference information. Let $X=\left\{x_{1}, x_{2}, \ldots, x_{n}\right\}$ be the set of alternatives, and $D=\left\{d_{1}, d_{2}, \ldots, d_{m}\right\}$ be the set of decision makers. Let $\lambda=\left(\lambda_{1}, \lambda_{2}, \ldots, \lambda_{m}\right)^{T}$ be the weight vector of decision makers, where $\lambda_{l} \geq 0, \sum_{l} \lambda_{l}=1$. The decision maker $d_{s} \in D$ compares these alternatives with respect to a single criterion by the multiplicative linguistic terms in the set $S=\left\{s_{\alpha} \mid \alpha=1 / t, \ldots, 1 / 2,1,2, \ldots, t\right\}$, and constructs the multiplicative linguistic preference relation $R^{(l)}=\left(r_{i j}^{(l)}\right)_{n \times n}$, whose element $r_{i j}^{(l)}$ estimates the preference degree of alternative $x_{i}$ over $x_{j}$, and meets

$$
s_{1 / t} \leq r_{i j}^{(l)} \leq s_{t}, r_{i j}^{(l)} \otimes r_{j i}^{(l)}=s_{1}, r_{i i}^{(l)}=s_{1} \text {, for all } i, j=1,2, \ldots, n
$$

It is well known that the multiplicative preference relations to express the judgements are reciprocal, however, In [14], Herrera and Herrera-Viedma showed that reciprocity generally is not preserved when aggregating multiplicative preference relations using the OWG operator. In the section, we shall show that the reciprocal property can be maintained when aggregating multiplicative linguistic preference relations using the EIOWG operator, where the order inducing variable $u_{l}=\lambda_{l}(l=1,2, \ldots, m)$.

Theorem 5: $\quad$ Let $R^{(1)}, R^{(2)}, \ldots, R^{(m)}$ be multiplicative linguistic preference relations provided by $m$ decision makers $d_{l}(l=1,2, \ldots, m)$, where $R^{(l)}=\left(r_{i j}^{(l)}\right)_{n \times n}, \quad r_{i j}{ }^{(l)} \in S \quad(l=1,2, \ldots, m ; i, j=1,2, \ldots, n)$, then their collective linguistic preference relation $\hat{R}=\left(\hat{r}_{i j}\right)_{n \times n}$ is also a multiplicative linguistic preference relation with $\hat{\mathrm{r}}_{\mathrm{ij}}=\mathrm{EIOWG}_{\mathrm{w}}\left(\left\langle\lambda_{1}, \mathrm{r}_{\mathrm{ij}}^{(1)}\right\rangle,\left\langle\lambda_{2}, \mathrm{r}_{\mathrm{ij}}^{(2)}\right\rangle, \ldots,\left\langle\lambda_{\mathrm{m}}, \mathrm{r}_{\mathrm{ij}}^{(\mathrm{m})}\right\rangle\right)=\left(\mathrm{b}_{\mathrm{ij}}^{(1)}\right)^{\mathrm{w}_{1}} \otimes\left(\mathrm{b}_{\mathrm{ij}}^{(2)}\right)^{\mathrm{w}_{2}} \otimes \ldots \otimes\left(\mathrm{b}_{\mathrm{ij}}^{(\mathrm{m})}\right)^{\mathrm{w}_{\mathrm{m}}}$ where $b_{i j}^{(k)}$ is the $r_{i j}^{(l)}$ value of the OWG pair $\left\langle\lambda_{l}, r_{i j}^{(l)}\right\rangle$ having the $k$ th largest $\lambda_{l}, s_{1 / t} \leq \hat{r}_{i j} \leq s_{t}, \quad \hat{r}_{i j} \otimes \hat{r}_{j i}=s_{1}, \hat{r}_{i i}=s_{1}$, for all $i, j=1,2, \ldots, n$.

Proof: Since $R^{(1)}, R^{(2)}, \ldots, R^{(m)}$ are multiplicative linguistic preference relations, we have $s_{1 / t} \leq r_{i j}^{(l)} \leq s_{t}$ and $r_{i j}^{(l)} \otimes r_{j i}^{(l)}=s_{1}, r_{i i}^{(l)}=s_{1}$, for all $l=1,2, \ldots, m ; i, j=1,2, \ldots, n$, and then

$$
\begin{aligned}
& \hat{\mathrm{r}}_{\mathrm{ij}}=\mathrm{EIOWG}_{\mathrm{w}}\left(\left\langle\lambda_{1}, \mathrm{r}_{\mathrm{ij}}^{(\mathrm{l})}\right\rangle,\left\langle\lambda_{2}, \mathrm{r}_{\mathrm{ij}}^{(2)}\right\rangle, \ldots,\left\langle\lambda_{\mathrm{m}}, \mathrm{r}_{\mathrm{ij}}^{(\mathrm{m})}\right\rangle\right)=\left(\mathrm{b}_{\mathrm{ij}}^{(1)}\right)^{\mathrm{w}_{1}} \otimes\left(\mathrm{b}_{\mathrm{ij}}^{(2)}\right)^{\mathrm{w}_{2}} \otimes \cdots \otimes\left(\mathrm{b}_{\mathrm{ij}}^{(\mathrm{m})}\right)^{\mathrm{w}_{\mathrm{m}}} \\
& \geq\left(s_{1 / t}\right)^{w_{1}} \otimes\left(s_{1 / t}\right)^{w_{2}} \otimes \ldots \otimes\left(s_{1 / t}\right)^{w_{m}}=\left(s_{1 / t}\right)^{\sum_{l=1}^{m} w_{l}}=s_{1 / t} \\
& \hat{r}_{i j}=E I O W G_{w}\left(\left\langle\lambda_{1}, r_{i j}^{(1)}\right\rangle,\left\langle\lambda_{2}, r_{i j}^{(2)}\right\rangle, \ldots,\left\langle\lambda_{m}, r_{i j}^{(m)}\right\rangle\right)=\left(b_{i j}^{(1)}\right)^{w_{1}} \otimes\left(b_{i j}^{(2)}\right)^{w_{2}} \otimes \ldots \otimes\left(b_{i j}^{(m)}\right)^{w_{m}}
\end{aligned}
$$




$$
\begin{aligned}
& \leq\left(s_{t}\right)^{w_{1}} \otimes\left(s_{t}\right)^{w_{2}} \otimes \cdots \otimes\left(s_{t}\right)^{w_{m}}=\left(s_{t}\right)^{\sum_{l=1}^{m} w_{l}}=s_{t} \\
& \hat{r}_{i j} \otimes \hat{r}_{j i}=\left(\left(b_{i j}^{(1)}\right)^{w_{1}} \otimes\left(b_{i j}^{(2)}\right)^{w_{2}} \otimes \ldots \otimes\left(b_{i j}^{(m)}\right)^{w_{m}}\right) \otimes\left(\left(b_{j i}^{(1)}\right)^{w_{1}} \otimes\left(b_{j i}^{(2)}\right)^{w_{2}} \otimes \cdots \otimes\left(b_{j i}^{(m)}\right)^{w_{m}}\right) \\
& =\left(\left(b_{i j}^{(1)} \otimes b_{j i}^{(1)}\right)^{w_{1}} \otimes\left(b_{i j}^{(2)} \otimes b_{j i}^{(2)}\right)^{w_{2}} \otimes \ldots \otimes\left(b_{i j}^{(m)} \otimes b_{j i}^{(m)}\right)\right)^{w_{m}} \\
& =\left(s_{1}\right)^{w_{1}} \otimes\left(s_{1}\right)^{w_{2}} \otimes \ldots \otimes\left(s_{1}\right)^{w_{m}}=\left(s_{1}\right)^{\sum_{l=1}^{m} w_{l}}=s_{1} \\
& \hat{r}_{i i}=\left(b_{i i}^{(1)}\right)^{w_{1}} \otimes\left(b_{i i}^{(2)}\right)^{w_{2}} \otimes \ldots \otimes\left(b_{i i}^{(m)}\right)^{w_{m}}=\left(r_{i i}^{(1)}\right)^{w_{1}} \otimes\left(r_{i i}^{(2)}\right)^{w_{2}} \otimes \cdots \otimes\left(r_{i i}^{(m)}\right)^{w_{m}} \\
& =\left(s_{1}\right)^{w_{1}} \otimes\left(s_{1}\right)^{w_{2}} \otimes \ldots \otimes\left(s_{1}\right)^{w_{m}}=\left(s_{1}\right)^{\sum_{l=1}^{m} w_{l}}=s_{1} \text { thus, } \hat{R} \text { is a multiplicative linguistic preference relation. }
\end{aligned}
$$

This completes the proof of Theorem 5 .

In the following, we shall apply the EIOWG and the EOWG operators to group decision making based on multiplicative linguistic preference relations.

Step 1: For a group decision making problem with linguistic preference information. The decision maker $d_{l} \in D$ compares these alternatives with respect to a single criterion by the multiplicative linguistic terms in $S$, and constructs the multiplicative linguistic preference relation $R^{(l)}=\left(r_{i j}^{(l)}\right)_{n \times n}$, where $s_{1 / t} \leq r_{i j}^{(l)} \leq s_{t}, r_{i j}^{(l)} \otimes r_{j i}^{(l)}=s_{1}, r_{i i}^{(l)}=s_{1}$, for all $i, j=1,2, \ldots, n$

Step 2: Utilize the EIOWG operator $\hat{r}_{i j}=E I O W G_{w}\left(\left\langle\lambda_{1}, r_{i j}^{(1)}\right\rangle,\left\langle\lambda_{2}, r_{i j}^{(2)}\right\rangle, \cdots,\left\langle\lambda_{m}, r_{i j}^{(m)}\right\rangle\right), i, j=1,2, \ldots, n$ to aggregate all the multiplicative linguistic preference relations $R^{(l)}=\left(r_{i j}^{(l)}\right)_{n \times n}(l=1,2, \ldots, m)$ to get the collective multiplicative linguistic preference relation $\hat{R}=\left(\hat{r}_{i j}\right)_{n \times n}$.

Step 3: Utilize the EOWG operator $\hat{r}_{i}=E O W G_{w}\left(\hat{r}_{i 1}, \hat{r}_{i 2}, \cdots, \hat{r}_{i n}\right), i=1,2, \ldots, n$ to aggregate $\hat{r}_{i j}(j=1,2, \ldots, n)$ corresponding to the alternative $x_{i}$, and then get the collective linguistic preference degree $\hat{r}_{i}(i=1,2, \ldots, n)$ of the $i$ th alternative over all the other alternatives.

Step 4: Rank all the alternatives and select the best one(s) in accordance with the values of $\hat{r}_{i}(i=1,2, \ldots, n)$.

\section{Step 5: End.}

Illustrative Example: Let us suppose an investment company, which wants to invest a sum of money in the best option (adapted from [32]). There is a panel with five possible alternatives in which to invest the money:

1) $x_{1}$ is a car industry; 2) $x_{2}$ is a food company; 3) $x_{3}$ is a computer company; 4) $x_{4}$ is an arms company;

5) $x_{5}$ is a TV company.

One main criterion used is growth analysis. There are three decision makers $d_{l}(l=1,2,3)$, whose weight vector 
$\lambda=(0.5,0.3,0.2)^{T}$. The decision makers compare these five companies with respect to the criterion growth analysis by using the multiplicative linguistic scale $\mathrm{S}=\left\{\mathrm{s}_{1 / 5}=\right.$ extremely low, $\mathrm{s}_{1 / 4}=$ very low, $\mathrm{s}_{1 / 3}=$ low, $s_{1 / 2}=$ slightly low, $s_{1}=$ medium, $s_{2}=$ slightly high, $\mathrm{s}_{3}=$ high, $\mathrm{s}_{4}=$ very high, $\mathrm{s}_{5}=$ extremely high $\}$

and construct, respectively, the multiplicative linguistic preference relations $R^{(l)} \quad(l=1,2,3)$ as listed in Tables $1-3$.

Table 1: Multiplicative Linguistic Preference Relation $R^{(1)}$

\begin{tabular}{llllll}
\hline & $x_{1}$ & $x_{2}$ & $x_{3}$ & $x_{4}$ & $x_{5}$ \\
\hline$x_{1}$ & $\mathrm{~s}_{1}$ & $\mathrm{~s}_{3}$ & $\mathrm{~s}_{1 / 4}$ & $\mathrm{~s}_{3}$ & $\mathrm{~s}_{1 / 4}$ \\
$x_{2}$ & $\mathrm{~s}_{1 / 3}$ & $\mathrm{~s}_{1}$ & $\mathrm{~s}_{4}$ & $\mathrm{~s}_{2}$ & $\mathrm{~s}_{1 / 3}$ \\
$x_{3}$ & $\mathrm{~s}_{4}$ & $\mathrm{~s}_{1 / 4}$ & $\mathrm{~s}_{1}$ & $\mathrm{~s}_{3}$ & $\mathrm{~s}_{5}$ \\
$x_{4}$ & $\mathrm{~s}_{1 / 3}$ & $\mathrm{~s}_{1 / 2}$ & $\mathrm{~s}_{1 / 3}$ & $\mathrm{~s}_{1}$ & $\mathrm{~s}_{4}$ \\
$x_{5}$ & $\mathrm{~s}_{4}$ & $\mathrm{~s}_{3}$ & $\mathrm{~s}_{1 / 5}$ & $\mathrm{~s}_{1 / 4}$ & $\mathrm{~s}_{1}$ \\
\hline
\end{tabular}

Table 2: Multiplicative Linguistic Preference Relation $R^{(2)}$

\begin{tabular}{llllll}
\hline & $x_{1}$ & $x_{2}$ & $x_{3}$ & $x_{4}$ & $x_{5}$ \\
\hline$x_{1}$ & $\mathrm{~s}_{1}$ & $\mathrm{~s}_{4}$ & $\mathrm{~s}_{1 / 3}$ & $\mathrm{~s}_{3}$ & $\mathrm{~s}_{1 / 2}$ \\
$x_{2}$ & $\mathrm{~s}_{1 / 4}$ & $\mathrm{~s}_{1}$ & $\mathrm{~s}_{4}$ & $\mathrm{~s}_{4}$ & $\mathrm{~s}_{1 / 3}$ \\
$x_{3}$ & $\mathrm{~s}_{3}$ & $\mathrm{~s}_{1 / 4}$ & $\mathrm{~s}_{1}$ & $\mathrm{~s}_{5}$ & $\mathrm{~s}_{4}$ \\
$x_{4}$ & $\mathrm{~s}_{1 / 3}$ & $\mathrm{~s}_{1 / 4}$ & $\mathrm{~s}_{1 / 5}$ & $\mathrm{~s}_{1}$ & $\mathrm{~s}_{5}$ \\
$x_{5}$ & $\mathrm{~s}_{2}$ & $\mathrm{~s}_{3}$ & $\mathrm{~s}_{1 / 4}$ & $\mathrm{~s}_{1 / 5}$ & $\mathrm{~s}_{1}$ \\
\hline
\end{tabular}

Table 3: Multiplicative Linguistic Preference Relation $R^{(3)}$

\begin{tabular}{llllll}
\hline & $x_{1}$ & $x_{2}$ & $x_{3}$ & $x_{4}$ & $x_{5}$ \\
\hline$x_{1}$ & $\mathrm{~s}_{1}$ & $\mathrm{~s}_{3}$ & $\mathrm{~s}_{1 / 2}$ & $\mathrm{~s}_{3}$ & $\mathrm{~s}_{1 / 5}$ \\
$x_{2}$ & $\mathrm{~s}_{1 / 3}$ & $\mathrm{~s}_{1}$ & $\mathrm{~s}_{5}$ & $\mathrm{~s}_{4}$ & $\mathrm{~s}_{1 / 4}$ \\
$x_{3}$ & $\mathrm{~s}_{2}$ & $\mathrm{~s}_{1 / 5}$ & $\mathrm{~s}_{1}$ & $\mathrm{~s}_{5}$ & $\mathrm{~s}_{3}$ \\
$x_{4}$ & $\mathrm{~s}_{1 / 3}$ & $\mathrm{~s}_{1 / 4}$ & $\mathrm{~s}_{1 / 5}$ & $\mathrm{~s}_{1}$ & $\mathrm{~s}_{4}$ \\
$x_{5}$ & $\mathrm{~s}_{5}$ & $\mathrm{~s}_{4}$ & $\mathrm{~s}_{1 / 3}$ & $\mathrm{~s}_{1 / 4}$ & $\mathrm{~s}_{1}$ \\
\hline
\end{tabular}

To get the most desirable alternative(s), the following steps are involved:

Step 1: Utilize the EIOWG operator (let its weighting vector be $\left.w=(0.3,0.4,0.3)^{T}\right)$

$$
\hat{r}_{i j}=E I O W G_{w}\left(\left\langle\lambda_{1}, r_{i j}^{(1)}\right\rangle,\left\langle\lambda_{2}, r_{i j}^{(2)}\right\rangle,\left\langle\lambda_{3}, r_{i j}^{(3)}\right\rangle\right), i, j=1,2,3,4,5
$$

to aggregate all the multiplicative linguistic preference relations $R^{(l)}=\left(r_{i j}^{(l)}\right)_{5 \times 5}(l=1,2,3)$

$$
\hat{r}_{11}=\operatorname{EIOWG}_{w}\left(\left\langle 0.5, r_{11}^{(1)}\right\rangle,\left\langle 0.3, r_{11}^{(2)}\right\rangle,\left\langle 0.2, r_{11}^{(3)}\right\rangle\right)=\left(s_{1}\right)^{0.3} \otimes\left(s_{1}\right)^{0.4} \otimes\left(s_{1}\right)^{0.3}=s_{1}
$$


Similarly, we have

$\hat{r}_{12}=s_{3.37}, \hat{r}_{13}=s_{0.35}, \hat{r}_{14}=s_{3}, \hat{r}_{15}=s_{0.31}$

$\hat{r}_{21}=s_{0.30}, \hat{r}_{22}=s_{1}, \hat{r}_{23}=s_{4.28}, \hat{r}_{24}=s_{3.25}, \hat{r}_{25}=s_{0.31}$

$\hat{r}_{31}=s_{2.90}, \hat{r}_{32}=s_{0.23}, \hat{r}_{33}=s_{1}, \hat{r}_{34}=s_{4.29}, \hat{r}_{35}=s_{3.92}$

$\hat{r}_{41}=s_{0.33}, \hat{r}_{42}=s_{0.31}, \hat{r}_{43}=s_{0.23}, \hat{r}_{44}=s_{1}, \hat{r}_{45}=s_{4.37}$

$\hat{r}_{51}=s_{3.24}, \hat{r}_{52}=s_{3.27}, \hat{r}_{53}=s_{0.26}, \hat{r}_{54}=s_{0.23}, \hat{r}_{55}=s_{1}$

and thus, we get the collective multiplicative linguistic preference relation $\hat{R}=\left(\hat{r}_{i j}\right)_{5 \times 5}$ :

Table 4: The Collective Preference Relation $\hat{R}$

\begin{tabular}{llllll}
\hline & $x_{1}$ & $x_{2}$ & $x_{3}$ & $x_{4}$ & $x_{5}$ \\
\hline$x_{1}$ & $\mathrm{~s}_{1}$ & $\mathrm{~S}_{3.37}$ & $\mathrm{~s}_{0.35}$ & $\mathrm{~s}_{3}$ & $\mathrm{~s}_{0.31}$ \\
$x_{2}$ & $\mathrm{~s}_{0.30}$ & $\mathrm{~S}_{1}$ & $\mathrm{~s}_{4.28}$ & $\mathrm{~s}_{3.25}$ & $\mathrm{~s}_{0.31}$ \\
$x_{3}$ & $\mathrm{~s}_{2.90}$ & $\mathrm{~S}_{0.23}$ & $\mathrm{~s}_{1}$ & $\mathrm{~s}_{4.29}$ & $\mathrm{~s}_{3.92}$ \\
$x_{4}$ & $\mathrm{~s}_{0.33}$ & $\mathrm{~S}_{0.31}$ & $\mathrm{~s}_{0.23}$ & $\mathrm{~s}_{1}$ & $\mathrm{~s}_{4.37}$ \\
$x_{5}$ & $\mathrm{~s}_{3.24}$ & $\mathrm{~S}_{3.27}$ & $\mathrm{~s}_{0.26}$ & $\mathrm{~s}_{0.23}$ & $\mathrm{~s}_{1}$ \\
\hline
\end{tabular}

Step 2: Utilize the EOWG operator (let its weighting vector be $\left.w=(0.1,0.2,0.4,0.2,0.1)^{T}\right)$

$\hat{r}_{i}=E O W G_{w}\left(\hat{r}_{i 1}, \hat{r}_{i 2}, \hat{r}_{i 3}, \hat{r}_{i 4}, \hat{r}_{i 5}\right)$ to aggregate $\hat{r}_{\mathrm{ij}}(\mathrm{j}=1,2,3,4,5)$ corresponding to the alternative $x_{i}$, and then get the collective linguistic preference degree $\hat{\mathrm{r}}_{\mathrm{i}}(\mathrm{i}=1,2,3,4,5)$ of the $i$ th alternative over all the other alternatives:

$$
\begin{aligned}
& \hat{r}_{1}=E O W G_{w}\left(\hat{r}_{11}, \hat{r}_{12}, \hat{r}_{13}, \hat{r}_{14}, \hat{r}_{15}\right) \\
& =\left(s_{3.37}\right)^{0.1} \otimes\left(s_{3}\right)^{0.2} \otimes\left(s_{1}\right)^{0.4} \otimes\left(s_{0.35}\right)^{0.2} \otimes\left(s_{0.31}\right)^{0.1}=s_{1.01} \\
& \hat{r}_{2}=E O W G_{w}\left(\hat{r}_{21}, \hat{r}_{22}, \hat{r}_{23}, \hat{r}_{24}, \hat{r}_{25}\right) \\
& =\left(s_{4.28}\right)^{0.1} \otimes\left(s_{3.25}\right)^{0.2} \otimes\left(s_{1}\right)^{0.4} \otimes\left(s_{0.31}\right)^{0.2} \otimes\left(s_{0.30}\right)^{0.1}=s_{1.03} \\
& \hat{r}_{3}=E O W G_{w}\left(\hat{r}_{31}, \hat{r}_{32}, \hat{r}_{33}, \hat{r}_{34}, \hat{r}_{35}\right) \\
& =\left(s_{4.29}\right)^{0.1} \otimes\left(s_{3.92}\right)^{0.2} \otimes\left(s_{2.90}\right)^{0.4} \otimes\left(s_{1}\right)^{0.2} \otimes\left(s_{0.23}\right)^{0.1}=s_{2.01} \\
& \hat{r}_{4}=E O W G_{w}\left(\hat{r}_{41}, \hat{r}_{42}, \hat{r}_{43}, \hat{r}_{44}, \hat{r}_{45}\right) \\
& =\left(s_{4.37}\right)^{0.1} \otimes\left(s_{1}\right)^{0.2} \otimes\left(s_{0.33}\right)^{0.4} \otimes\left(s_{0.31}\right)^{0.2} \otimes\left(s_{0.23}\right)^{0.1}=s_{0.51} \\
& \hat{r}_{5}=E O W G_{w}\left(\hat{r}_{51}, \hat{r}_{52}, \hat{r}_{53}, \hat{r}_{54}, \hat{r}_{55}\right)
\end{aligned}
$$


$=\left(s_{3.27}\right)^{0.1} \otimes\left(s_{3.24}\right)^{0.2} \otimes\left(s_{1}\right)^{0.4} \otimes\left(s_{0.26}\right)^{0.2} \otimes\left(s_{0.23}\right)^{0.1}=s_{0.94}$

thus, we have $\hat{r}_{3}>\hat{r}_{2}>\hat{r}_{1}>\hat{r}_{5}>\hat{r}_{4}$

Step 3: Rank all the alternatives in accordance with the values of $\hat{r}_{i} \quad(i=1,2,3,4,5)$ :

$x_{3} \succ x_{2} \succ x_{1} \succ x_{5} \succ x_{4}$ and thus the most desirable alternative is $x_{3}$.

\section{CONCLUSION}

In this study, we have developed an extended induced ordered weighted geometric (EIOWG) operator, which takes as its argument pairs, called OWG pairs, in which one component is used to induce an ordering over the second components which are linguistic variables. We have studied some desirable properties of the EIOWG operator, and then applied the EIOWG operator to group decision making based on multiplicative linguistic preference relations. In the future, we shall continue working in the application of the EIOWG operator to other domains.

\section{ACKNOWLEDGEMENT}

This study was supported by China Postdoctoral Science Foundation (2003034366).

\section{REFERENCES}

1. Xu, Z.S. and Q.L. Da, 2003. An overview of operators for aggregating information. Int. J. Intelligent Systems, 18: 953-969.

2. Yager, R.R., 1988. On ordered weighted averaging aggregation operators in multicriteria decision making. IEEE Transactions on Systems, Man and Cybernetics, 18: 183-190.

3. Yager, R.R., 1993. Families of OWA operators. Fuzzy Sets and Systems, 59: 125-148.

4. Yager, R.R. and J. Kacprzyk, 1997. The ordered weighted averaging operators: theory and applications. Norwell, MA: Kluwer.

5. Torra, V., 1997. The weighted OWA operator. Intelligent J. Intelligent Systems, 12: 153-166.

6. Mitchell, H.B., D.D. Estrakh, 1998. An OWA operator with fuzzy ranks. Intelligent J. Intelligent Systems, 13: 69-81.

7. Schaefer, P.A. and H.B. Mitchell, 1999. A generalized OWA operator. Int. J. Intelligent Systems, 14: 123-143.

8. Xu, Z.S. and Q.L. Da, 2002. The uncertain OWA Operator. Int. J. Intelligent Systems, 17: 569-575.
9. Chiclana, F., F. Herrera and E. Herrera-Viedma, 2000. The ordered weighted geometric operator: Properties and application. In Proc $8^{\text {th }}$ Int. Conf. on Information Processing and Management of Uncertainty in Knowledge-based Systems. Madrid, Spain, pp: 985-991.

10. Chiclana, F., F. Herrera and E. Herrera-Viedma, 2001. Integrating multiplicative preference relations in a multipurpose decision making model based on fuzzy preference relations. Fuzzy Sets and Systems, 112: 277-291.

11. Herrera, F., E. Herrera-Viedma and F. Chiclana, 2001. Multiperson decision making based on multiplicative preference relations. Eureapon J. Oper. Res., 129: 372-385.

12. Xu, Z.S. and Q.L. Da, 2002. The ordered weighted geometric averaging operators. Int. J. Intelligent Systems, 17: 709-716.

13. Xu, Z.S., 2002. Study on methods for multiple attribute decision making under some situations. Ph.D Thesis. Southeast University, Nanjing, China.

14. Herrera, F., E. Herrera-Viedma and F. Chiclana, 2003. A study of the origin and uses of the ordered weighted geometric operator in multicriteria decision making. Int. J. Intelligent Systems, 18: 689-707.

15. Xu, Z.S. and Q.L. Da, 2004. An uncertain ordered weighted geometric (UOWG) operator and its application. Information: An Int. J., 7: 175-182.

16. Yager, R.R. and D.P. Filev, 1999. Induced ordered weighted averaging operators. IEEE Transactions on Systems Man, and Cybernetics-Part B, 29: 141-150.

17. Chiclana, F., E. Herrera-Viedma, F. Herrera and S. Alonso, 2004. Induced ordered weighted geometric operators and their use in the aggregation of multiplicative preference relations, Intl. J. Intelligent Systems 19: 233-255.

18. Zadeh, L.A., 1976. The concept of a linguistic variable and its application to approximate reasoning. Part 1,2 and 3, Information Sciences, 8: 199-249, 301-357, 1975. 9: 43-80, 1976. 
19. Tong, R. and P.P. Bonissone, 1980. A linguistic approach to decision making with fuzzy sets. IEEE Transactions on Systems, Man and Cybernetics, 10: 716-723.

20. Zadeh, L.A., 1983. A computational approach to fuzzy quantifiers in natural languages. Comp. Math. Appl., 9: 149-184.

21. Kacprzyk, J., 1986. Group decision making with a fuzzy linguistic majority. Fuzzy Sets and Systems, 18: 105-118.

22. Degani, R. and G. Bortolan, 1988. The problem of linguistic approximation in clinical decision making. Int. J. Approximate Reasoning, 2: 143-162.

23. Yager, R.R., 1993. Non-numeric multi-criteria multi-person decision making. Group Decision Negotiation, 2: 81-93.

24. Delgado, M., J.L. Verdegay and M.A. Vila, 1993. On aggregation operations of linguistic labels. Int. J. Intelligent Systems, 8: 351-370.

25. Herrera, F., 1995. A sequential selection process in group decision making with linguistic assessment. Information Sciences, 85: 223-239.

26. Torra, V., 1996. Negation functions based semantics for ordered linguistic labels. Int. J. Intelligent Systems, 11: 975-988.

27. Bordogna, G., M. Fedrizzi and G. G. Passi, 1997. A linguistic modeling of consensus in group decision making based on OWA operator. IEEE Transactions on Systems, Man, and Cybernetics, 27: 126-132.

28. Zadeh, L.A. and J. Kacprzyk, 1999. Computing with words in information/intelligent systems-part 1: foundations: part 2: applications. Heidelberg, Germany: Physica-Verlag, vol. I.

29. Herrera, F. and E. Herrera-Viedma, 2000. Choice functions and mechanisms for linguistic preference relations. European J. Oper. Res., 120: 144-161.
30. Herrera, F. and L. Martínez, 2000. An approach for combining linguistic and numerical information based on 2-tuple fuzzy linguistic representation model in decision-making. Int. J. Uncertainty, Fuzziness, Knowledge-based Systems, 8: 539-562.

31. Herrera, F. and L. Martínez, 2000. A 2-tuple fuzzy linguistic representation model for computing with words. IEEE Tran. fuzzy systems, 8: 746-752.

32. Herrera, F., E. Herrera-Viedma and L. Martínez, 2000. A fusion approach for managing multigranularity linguistic term sets in decision making. Fuzzy Sets and Systems, 114: 43-58.

33. Herrera, F. and L. Martínez, 2001. A model based on linguistic 2-tuples for dealing with multigranular hierarchical linguistic contexts in multi-expert decision-making. IEEE Trans. Systems, Man, and Cybernetics, 31: 227-234.

34. Torra, V., 2001. Aggregation of linguistic labels when semantics is based on antonyms. Int. J. Intelligent Systems, 16: 513-524.

35. Cordón, O., F. Herrera and I. Zwir, 2002. Linguistic modeling by hierarchical systems of linguistic rules. IEEE Transactions on fuzzy systems, 10: 2-20.

36. Xu, Z.S., 2004. A method based on linguistic aggregation operators for group decision making with linguistic preference relations. Inform. Sci., 166: 19-30.

37. Xu, Z.S., 2004. A method based on linguistic aggregation operators for group decision making with linguistic preference relations. Information Sciences, 166: 19-30.

38. Xu, Z.S., 2004. Uncertain multiple attribute decision making: methods and applications. Beijing: Tsinghua University Press, China. 